\title{
Deterministic Transfer of Large-Scale $\beta$-Phase Arsenic on Fiber End Cap for Near-Infrared Ultrafast Pulse Generation
}

\author{
Qiang $Y u^{1,2}$, Cheng Chen $^{2}$, Kun Guo ${ }^{1}$, Haiqin Deng ${ }^{1}$, Tianan $Y_{i}{ }^{3}$, Yan Zhang ${ }^{2}$, Wei Su ${ }^{3,4}$, \\ Jian $\mathrm{Wu}^{1 *}$ and Kai Zhang ${ }^{2 *}$ \\ ${ }^{1}$ College of Advanced Interdisciplinary Studies, National University of Defense Technology, Changsha, China, ${ }^{2}$-Lab \& Key \\ Laboratory of Nanodevices and Applications \& Key Laboratory of Nanophotonic Materials and Devices, Suzhou Institute of Nano- \\ Tech and Nano-Bionics, Chinese Academy of Sciences, Suzhou, China, ${ }^{3}$ College of Mechanical and Electrical Engineering, Hohai \\ University, Changzhou, China, ${ }^{4}$ College of Science, Hohai University, Changzhou, China
}

OPEN ACCESS

Edited by:

Qiaoliang Bao,

Soochow University, China

Reviewed by:

Xiaohui Li,

Shaanxi Normal University, China

M. Sasani Ghamsari,

Atomic Energy Organization of

Iran, Iran

Haoran $\mathrm{Mu}$,

Songshan Lake Material Laboratory,

China

*Correspondence:

Jian Wu

wuijan15203@163.com

Kai Zhang

kzhang2015@sinano.ac.cn

Specialty section:

This article was submitted to

Thin Solid Films,

a section of the journal

Frontiers in Materials

Received: 07 June 2021

Accepted: 02 August 2021

Published: 17 September 2021

Citation:

Yu Q, Chen C, Guo K, Deng H, Yi T, Zhang $Y$, Su W, Wu J and Zhang $K$ (2021) Deterministic Transfer of LargeScale $\beta$-Phase Arsenic on Fiber End

Cap for Near-Infrared Ultrafast

Pulse Generation.

Front. Mater. 8:721587.

doi: 10.3389/fmats.2021.721587
Gray arsenic ( $\beta$-phase) has aroused great attention in photonics and electronics applications, as a novel family member of two-dimensional (2D) elemental crystals of group-VA. Here, $\beta$-phase arsenic ( $\beta$-As) bulk crystals were synthesized via the chemical vapor transport (CVT) method. Meanwhile, large-scale $\beta$-As nanoflake was transformed using the polydimethylsiloxane (PDMS)-assisted dry transfer method and was placed on the end cap of optical fiber with high coverage over the core area. Moreover, the $\beta$-As was used as a saturable absorber in ytterbium-doped fiber ring cavity resonance, and we demonstrated near-infrared ultrafast pulse fiber laser with the central wavelength, repetition rate, and signal-to-noise ratio (SNR) of $1,037.3 \mathrm{~nm}, 0.6 \mathrm{MHz}$, and $67.7 \mathrm{~dB}$, respectively. This research demonstrates a $2 \mathrm{D}$ material small area deterministic transfer method and promotes the potential application of group-VA crystals in near-infrared ultrafast laser generation.

Keywords: $\beta$-phase arsenic, ultrafast pulse generation, near-infrared, CVT, nonlinear optics

\section{INTRODUCTION}

Two-dimensional (2D) materials have attracted various interests since the first discovery of monolayer graphene in 2004 and have shown great potential in near-infrared ultrashort pulse fiber laser generation. In the past decade, facing wide range application requirements of near-infrared ultrashort pulse (Jackson, 2012; Geng et al., 2014), such as LIDAR (Tang et al., 2016), optical communications (Moulton et al., 2009; Soref, 2015), material science (Salam et al., 2021; Shaodong et al., 2021), mid-infrared laser sources (Pawliszewska et al., 2017; Tuo et al., 2018), spectroscopy (Donodin et al., 2020; Xu N. et al., 2020), and biomedicine (Chen et al., 2019; Martov et al., 2020), a series of 2D material SA have been realized for pulse fiber laser generation. The applications in increasingly extensive fields demand the discovery of novel 2D material SA with particular properties. During the past years, various novel 2D material SA have been used for pulse fiber laser generation, including graphene (Martinez and Sun, 2013; Meng et al., 2015), topological insulators (TI) (Bao et al., 2009; Lee et al., 2014; Liu et al., 2014; Wang et al., 2021), black phosphorus (BP) (Ma et al., 2015; Hisyam et al., 2017), and transition metal dichalcogenides (TMDCs) (Gillen and Maultzsch, 2017; Tuo et al., 2018) (Wang J. et al., 2019; Shi et al., 2019; Chen et al., 2021), for the benefit of adjustable nonlinear absorption coefficient, short recovery time, and low optical lose. 

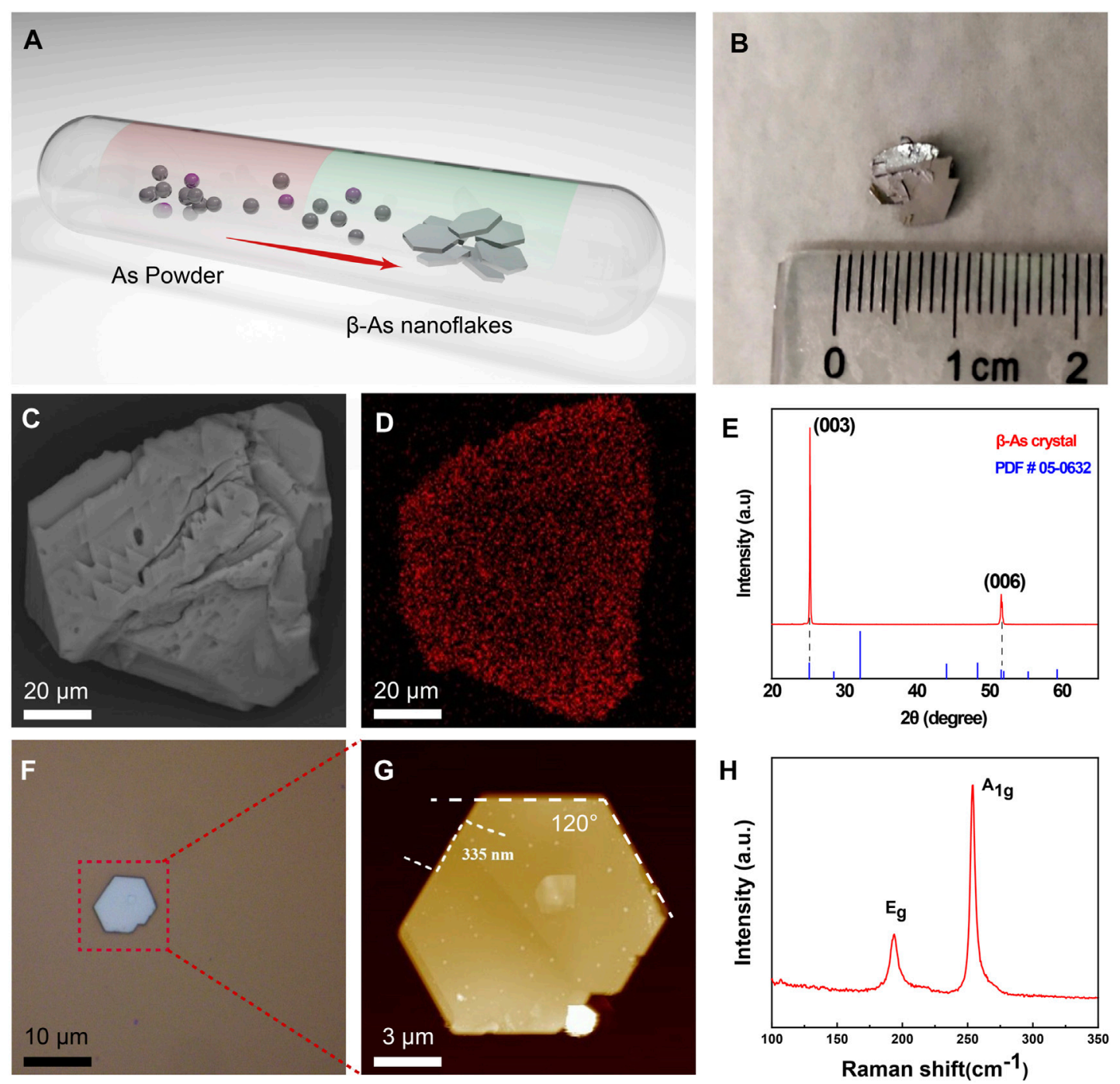

FIGURE 1 | $\beta$-phase arsenic crystals synthesis and characteristics. (A) Schematic illustration of the growth process of $\beta$-As crystals via the CVT method. (B) The optical image of $\beta$-As single crystal besides a ruler. (C) SEM and (D) the energy dispersive X-ray (EDX) mapping of $\beta$-As crystal. (D) The optical image of the typical exfoliated nanoflake after transferred on the SiO2/Si substrate. (E) X-ray diffraction (XRD) spectrum of $\beta$-As crystal. (F) A $\beta$-As nanoflake transferred on SiO2/Si substrate. (G) AFM image and the corresponding height of $\beta$-As nanoflake. (H) Raman spectrum of the exfoliated $\beta$-As flake obtained with a $532 \mathrm{~nm}$ laser at about $300 \mathrm{~K}$.

With the rapid development of novel $2 \mathrm{D}$ material preparation, at the same time, arsenic compounds (gallium arsenide, cadmium arsenide, and black arsenic phosphorus) are rising in industry and scientific research (Yoon et al., 2010; Zhang C. et al., 2019; Khalatpour et al., 2021). The raw material arsenic is widely used in many fields due to its excellent physical and chemical properties and has been thoroughly studied and recognized as building blocks for future photons and optoelectronic technologies. Along with the inspiration of the research on few-layer phosphorous allotrope, the few-layer arsenic allotrope is also concerned. They are both elemental layered materials derived from group VA (phosphorus, arsenic, antimony (Ji et al., 2016; Wu et al., 2017), and bismuth) (Zhang S. et al., 2016; Zhang et al., 2018; Niu et al., 2019; Wu and Hao, 2020). BP shows high mobility of up to about $1,000 \mathrm{~cm}^{2} \mathrm{~V}^{-1} \mathrm{~s}^{-1}$ and is used in nano-electronic and photonic devices, while it is very unstable and degrades rapidly in ambient conditions (Wang et al., 2019b; Xu et al., 2020b). Purple phosphorus with a pyrolysis temperature above $512^{\circ} \mathrm{C}$ is the most stable phosphorus allotrope (Zhang L. et al., 2020), and for blue phosphorus, an indirect band gap semiconductor (Zhang J. L. et al., 2016; Zhang J. L. et al., 2020), the photoelectric response can reach the ultraviolet region. The different properties of materials corresponding to different structures have aroused people's interest in arsenic research. The research on few-layer a-As (black arsenic) started in 2018 (Zhong et al., 2018). The $\beta$-As (gray arsenic) thin film has also been developed in recent years. It has the same structure as blue phosphorus, which displays rhombohedral stacking of layers (Zhao et al., 2017). The wide bandgap $(0-1.71 \mathrm{ev})$ with adjustable layers are 
a

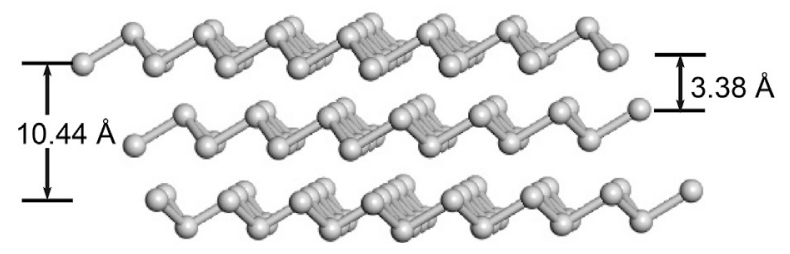

b

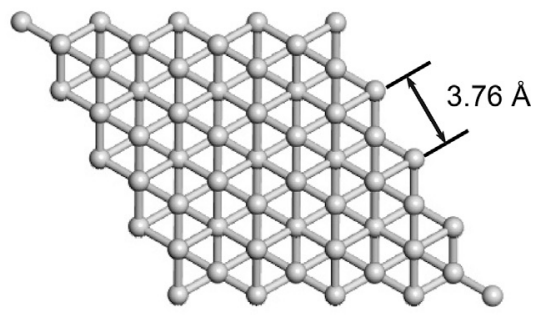

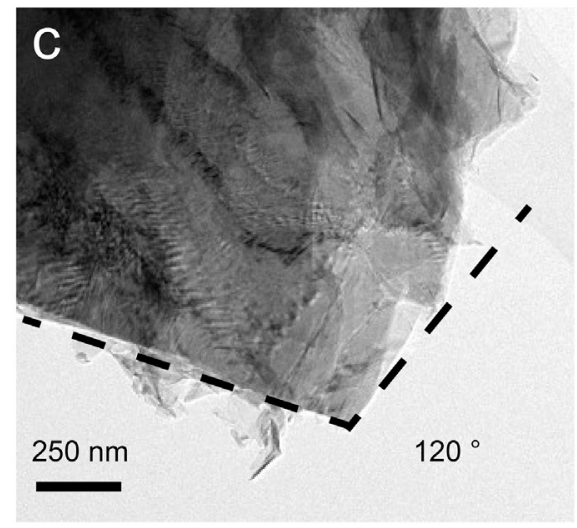
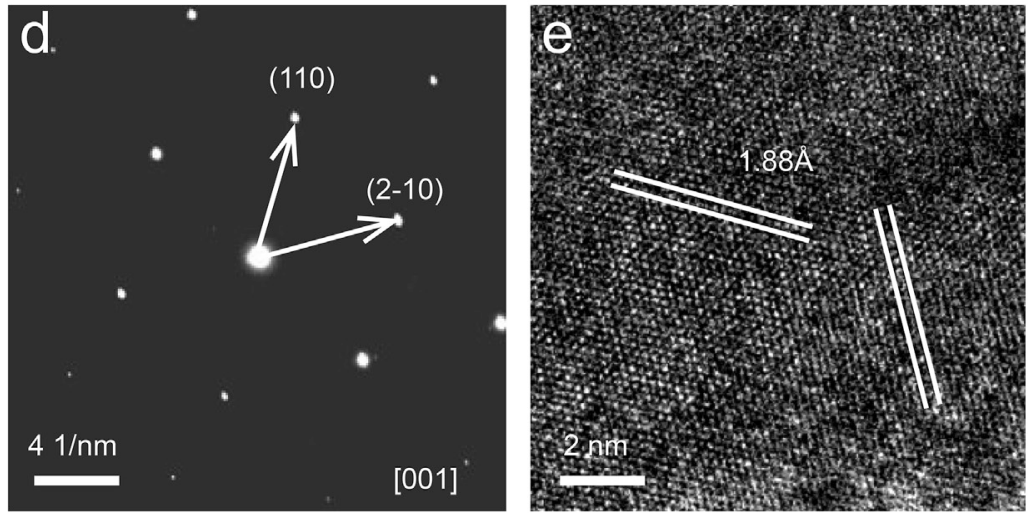

FIGURE 2 | Crystal structure of the $\beta$-phase arsenic flakes. (A) Buckled honeycomb structure of $\beta$-As. (B) Top view of the buckled arsenene. (C) The morphology of $\beta$-As nanoflake at low magnification. (D) The selected area electron diffraction (SAED) pattern. (E) The high-resolution TEM (HRTEM) image of a representative $\beta$-As nanoflake.

predicted in some theoretical articles (Kamal and Ezawa, 2015; Zhou et al., 2017). The higher carrier mobility in bulk gray arsenic has also been observed in few-layer arsenic (Hu et al., 2019). There are signs that $\beta$-As may become an excellent contender for a new generation of $2 \mathrm{D}$ nano-electronic, photonics devices. In addition, to obtain ultrashort pulse laser with a high signal-to-noise ratio and long-term stability, the quality of saturable absorber (SA) is a key component (Guo et al., 2015; Hu et al., 2018; Wang et al., 2019c; Zhang M. et al., 2019). Researchers are looking for reliable ways to place the nanomaterials at the fiber core, such as a special platform with a small area. The scotch tape-assisted approach, light-induced deposition method, end-to-end self-assembly, and embedding the layered materials in transparent polymer were reported (Lee et al., 2016; Rusdi et al., 2016; Hu et al., 2017; Cuando-Espitia et al., 2019). However, nanoflake accurate transfer is still a huge challenge.

In this study, $\beta$-phase arsenic ( $\beta$-As) bulk crystals were synthesized via the CVT method, and the morphology and structure were studied. With our PDMS-assisted accurate positioning dry transfer method, the gray arsenic nanoflake saturable absorber was prepared on the end cap of optical fiber with $100 \%$ yield. The g-As nanoflake-based ytterbiumdoped fiber laser can realize a stable mode-locked pulse with the central wavelength, repetition rate, and signal-to-noise ratio (SNR) of $1,037.3 \mathrm{~nm}, 0.6 \mathrm{MHz}$, and $67.7 \mathrm{~dB}$, respectively.

\section{SAMPLE PREPARATION AND CHARACTERIZATIONS}

\section{Growth Method}

$\beta$-As crystalline bulks were synthesized by using the CVT method (Xu et al., 2020c) in a furnace with two temperature zones, as schematically shown in Figure 1A, where the red area indicates the high temperature zone and the green area indicates the low temperature zone. Growth processes of $\beta$-As crystal are as follows: gray arsenic powders were used as precursor. First of all, $15 \mathrm{mg}$ gray arsenic powder was put into a quartz tube (length of $120 \mathrm{~mm}$, diameter of $20 \mathrm{~mm})$ and subsequently sealed up under vacuum $(<1 \times$ $\left.10^{-2} \mathrm{~Pa}\right)$. Afterward, the as-sealed quartz tube was placed in the furnace (OTF-1200X), and the temperature program of high temperature zone was set as a program curve: heating up to $500^{\circ} \mathrm{C}$ within $5 \mathrm{~h}$, keeping at $500^{\circ} \mathrm{C}$ for $1 \mathrm{~h}$, and then cooling down to room temperature in $5 \mathrm{~h}$. Finally, the product was obtained at the low temperature zone.

\section{Characterization Apparatus}

The morphology of the samples was investigated by optical microscopy (Nikon Eclipse LV100ND microscope) and atomic force microscopy (AFM, Bruker Dimension Icon 3,100). Scanning electron microscopy (SEM) and the corresponding energy-dispersive X-ray spectroscopy (EDX) characterizations 


\section{A}

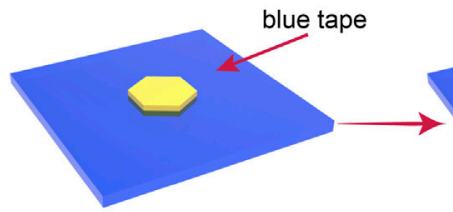

(1)

$\beta$-As crystal

Mechanical exfoliation with bule tape

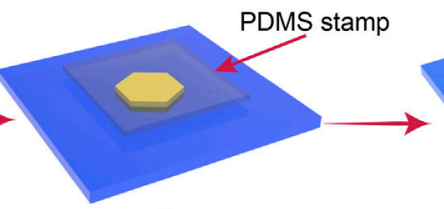

(2)

$\beta$-As nanoflake adhere

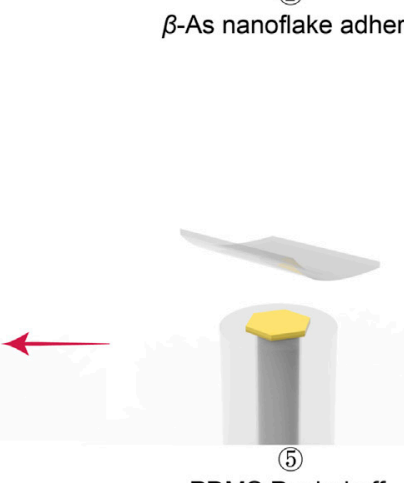

PDMS Peeled off
Deterministic Transfered sample

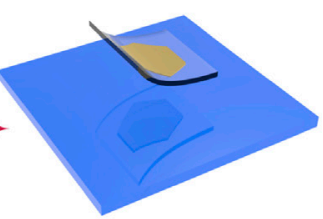

(3)

$\beta$-As nanoflake lifted with PDMS

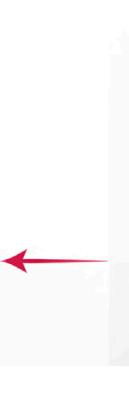

Selected $\beta$-As nanoflake alignment
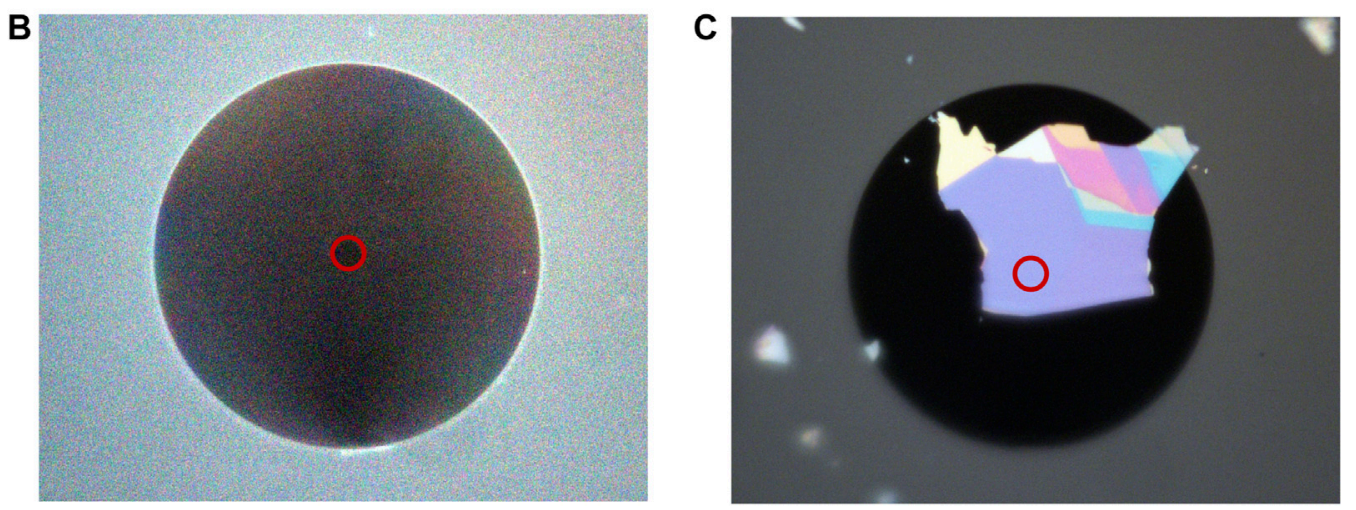

FIGURE 3 |Deterministic transfer of $\beta$-As nanoflake on fiber end cap. (A) The protocol of deterministic transfer: step 1, $\beta$-As bulk crystal mechanical exfoliation with blue tape; step 2, $\beta$-As nanoflake adhere; step 3, $\beta$-As nanoflake lifted with PDMS; step 4, the selected $\beta$-As nanoflake alignment; step 5 , PDMS peeled off; step 6 , deterministic transferred sample of $\beta$-As nanoflake. (B) Microscope digital image of the fiber end cap (diameter of red circle is $9 \mu \mathrm{m}$ ) before transfer. (C) Microscope digital image of fiber end cap after deterministic transfer of $\beta$-As nanoflake.

were carried out on a SEM (Quanta FEG 250) instrument, with an operating voltage of $30 \mathrm{kV}$ and a spot line of 5.0. Raman measurements were performed in a confocal Raman spectrometer (Renishaw inVia), with an excitation laser of $532 \mathrm{~nm}$ wavelength and a $\times 100$ objective lens. X-ray diffraction (XRD) was performed with a powder X-ray diffractometer (Bruker AXS D8 Advance) system with $\mathrm{Cu} \mathrm{Ka}$ irradiation $(\lambda=1.5406 \AA)$. Transmission electron microscopy (TEM) and selected area electron diffraction (SAED) were performed on a TEM (Tecnai G2 F20 S-TWIN) instrument.

\section{Characterization and Analysis}

As shown in the optical image (Figure 1B), the $\beta$-As single crystal is about $8 \mathrm{~mm}$ size, and obvious geometric corners are found on it. Its luminal appearance indicates that thickness can be reduced by mechanical stripping. SEM with EDX measurements was conducted to observe the morphology and analyze the chemical element of the $\beta$-As crystals. Figure 1C shows a flake with regular edges and corners. The EDX mapping verified the uniform distribution of unique arsenic element, as shown in Figure 1D. The powder XRD was applied to judge the crystal structure and the phase purity of the as-synthesized $\beta$-As crystals. In Figure 1E, the peaks match with the rhombohedral structure in space group R3m (PDF \# 05-0,632) (Hu et al., 2019). The diffraction peak at about $25.28^{\circ}$ and $52.04^{\circ}$ can be well-indexed to the (003) and (006) plane, respectively. What is more, the XRD pattern demonstrates that the $\beta$-As crystals present a highly preferred orientation along the $(00 L)$ direction. Figure 1F shows a typical exfoliated $\beta$-As nanoflake after it was transferred onto the $\mathrm{SiO} 2 / \mathrm{Si}$ substrate. It can be seen that the surface is uniform without impurities. The corresponding atomic force microscopy (AFM) image is shown in 
A

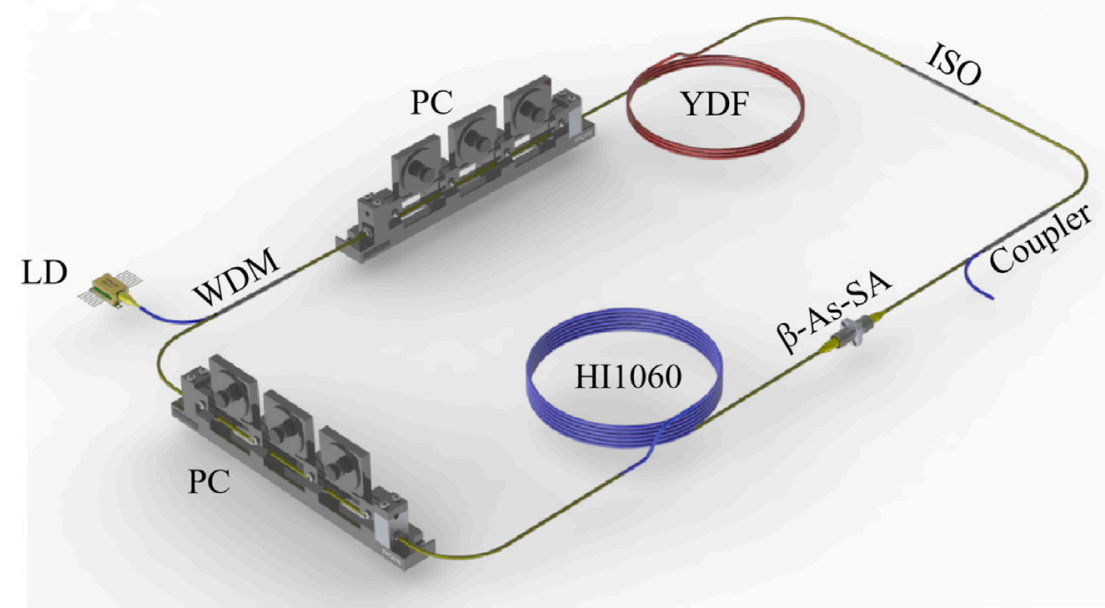

B

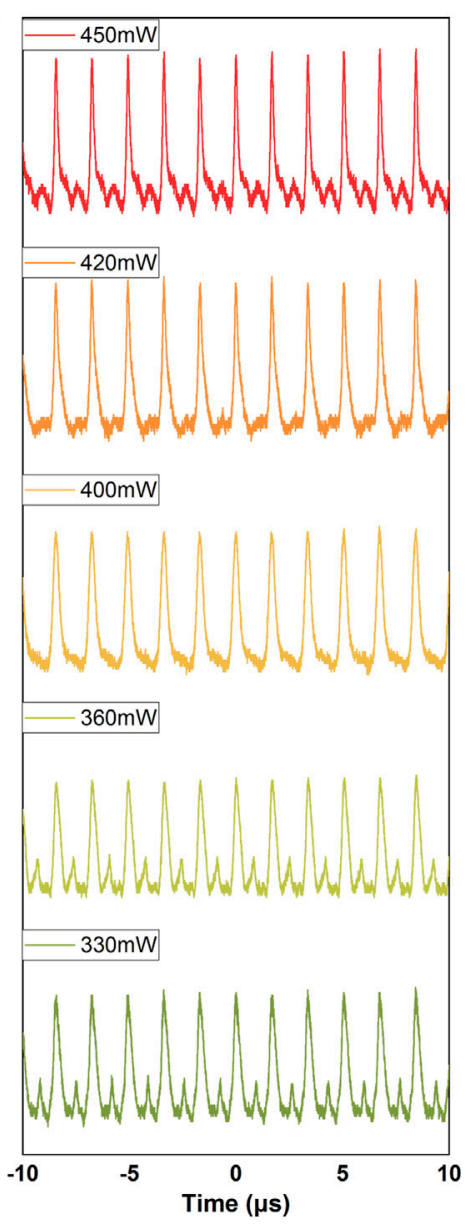

C

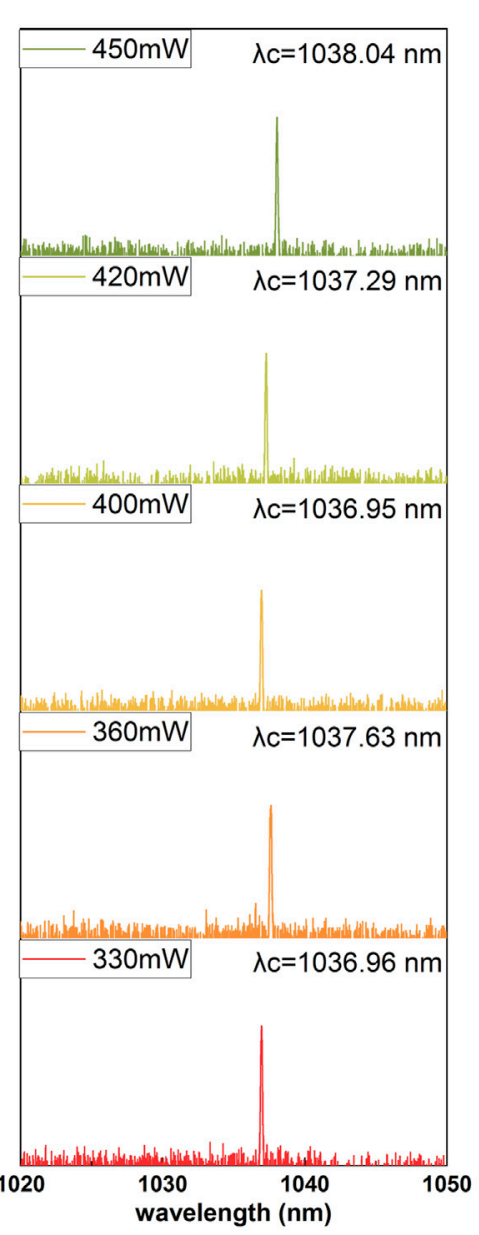

D

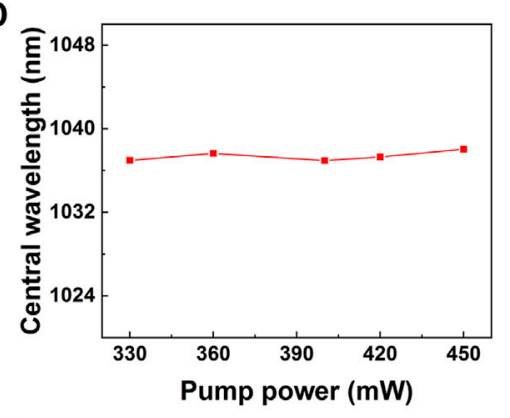

E

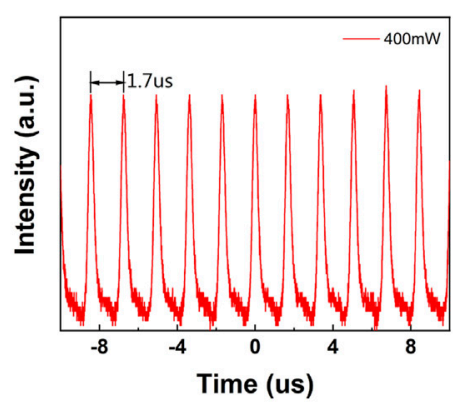

$\mathbf{F}$

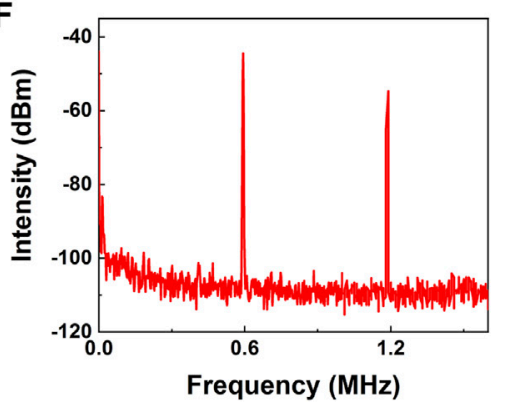

FIGURE 4 | Pulse generation from the Yb-doped fiber ring cavity based on the $\beta$-As SA. (A) Schematic of the all-fiber 1- $\mu$ m pulse laser based on $\beta$-As SA. (B) Pulse sequences at different pump powers. (C) Normalized emission spectra at different pump powers. (D) Central wavelength versus incident pump power. (E) Pulse interval at $400 \mathrm{mw}$. (F) Radio frequency (RF) spectrum. 
Figure 1G. It has a regular angle of $120^{\circ}$, and its height is about $335 \mathrm{~nm}$. It is relatively difficult to exfoliate the $\beta$-As bulk to very thin flakes, which may result from a strong interlayer interaction. Raman spectrum was carried out as fingerprint authentication of materials. As shown in Figure $\mathbf{1 H}$, two characteristic peaks with frequencies of 195.3 and $254.5 \mathrm{~cm}^{-1}$ are corresponding to $\mathrm{E}_{\mathrm{g}}$ (in-plane vibration) and $\mathrm{A}_{1 \mathrm{~g}}$ (out-ofplane vibration) modes of $\beta$-As, respectively.

In order to further discuss the crystal structure, we investigated $\beta$-As with a rhombohedral A7 structure (Zhu et al., 2015). The crystal is a double-layered structure composed of many interlocking six-membered rings. The crystal structure model of the $\beta$-As was observed in different views in Figures 2A,B. It is a kind of buckled $2 \mathrm{D}$ hexagonal structure, with a lattice constant of $\mathrm{a}=3.76 \AA$, $\mathrm{c}=10.44 \AA$, and As-As bond length of $2.5 \AA$. Afterward, the $\beta$-As nanoflakes were transferred to the $\mathrm{Cu}$ grid for transmission electron microscopy characterizations. Figure 2B illustrates a hexagonal $\beta$-As nanoflake with an angle of $120^{\circ}$. As shown in Figure 2C, the electron diffraction pattern presents a refined hexagonal structure, which is consistent with the XRD data (PDF \# 05-0,632). Figure 2D shows the high-resolution TEM (HRTEM) characterization of the same $\beta$-As nanoflake with distinct lattice fringes without obvious impurities. The lattice fringes marked in the figure shows a fixed spacing, which is measured as $1.88 \AA$. These crystal planes can be indexed to (110) and (2-10), with the zone axis of [001] orientation.

\section{Deterministic Transfer on Fiber End Face}

$\beta$-As nanoflake was accurately transferred onto a fiber facet as an SA by the homemade 3D transfer platform (Figure 3). The protocol schematic of deterministic transfer was shown in Figure 3A. The specific dry transfer process is described as follows: First of all, the $\beta$-As bulk was thinned by mechanical exfoliation with a blue tape. Homemade polydimethylsiloxane (PDMS) stamp was used to adhere thinned $\beta$-As nanoflakes. As the PDMS is transparent, the thickness and size of $\beta$-As nanoflake can be roughly determined through it. When a suitable flake has been identified, the underlying fiber end face is fixed on the sample stage. The PDMS with target nanoflake is then fixed to the three-axis cantilever with the flakes facing the fiber core. When the PDMS and the fiber core are close to the focal plane of the microscope, it is possible to align the right flake toward the fiber core. Finally, the stamp is pressed against the fiber end face and peeled off very slowly. As shown in Figure 3B, the fiber core (diameter: $9 \mu \mathrm{m}$ ) was marked by a red circle in the dark field optical microscope image. After transfer, the fiber core was completely covered with a $\beta$-As nanoflake (Figure 3C). In this way, the resulting nanoflakes have no bulges or wrinkles.

\section{PULSE GENERATION AND DISCUSSION}

The sandwiched structure $\beta$-As-SA was installed into the laser ring cavity. As illustrated in Figure 4A, the total length of the ring cavity is about $351 \mathrm{~m}$. A laser diode (LD) operating at $976 \mathrm{~nm}$ wavelength was used to pump a 1.5-m-long ytterbium-doped fiber (YDF) (6/125) through a 980/1,060 $\mathrm{nm}$ wavelength division multiplexing (WDM). In order to realize unidirectional waveguide of the laser, a polarization-independent isolator (PIISO) was connected with the YDF. The SA device of sandwiched structure was placed between the optical coupler (OC) and 300-m single-mode fiber (SMF) (HI1060). We adopted two polarization controllers (PC) to adjust the phase of the laser oscillation mode. Besides, we used an OC of 20:80 ratio which has a $1 \times 2$ pigtail structure. The $20 \%$ laser output was separated from the laser cavity to measure the laser characteristics by the oscilloscope or optical spectrum analyzer (OSA). The remaining 80\% laser was coupled into the laser cavity to form laser oscillation.

By increasing the pump power to $330 \mathrm{~mW}$ and adjusting the PC appropriately, the mode-locked pulse phenomenon took place. The state was not very stable at the beginning of the startup, and the pulse splits slightly. However, it kept an obvious pulse interval under $330-450 \mathrm{~mW}$, as shown in Figure 4B. The corresponding output spectrum is illustrated in Figure 4C, with a slight change in the central wavelength of around 1,037 $\mathrm{nm}$ (Figure 4D). The output pulse trace is shown in Figure 4E, with a pulse interval of $1.7 \mu$ s under $400 \mathrm{~mW}$. The corresponding radio frequency (RF) spectrum is as shown in Figure 4F. The signal-to-noise ratio (SNR) is about $58.5 \mathrm{~dB}$, and the repetition rate is $0.6 \mathrm{MHz}$. In the 3-day sampling period, the pulse sequence and spectral output are basically stable.

\section{CONCLUSION}

In conclusion, high-quality $\beta$-As were successfully synthesized, and an ytterbium-doped fiber mode-locked laser based on $\beta$-AsSA has been realized for the first time. A relative stable $1-\mu \mathrm{m}$ pulsed laser was generated at a pump power ranging from 330 to $450 \mathrm{~mW}$ with an almost unchanged repetition rate of $0.6 \mathrm{MHz}$ and a central wavelength around $1,037 \mathrm{~nm}$. This work demonstrates a 2D material small area deterministic transfer method and promotes the research of near-infrared pulsed lasers based on 2D materials and also shows the great potential of group VA crystals for nonlinear photonic applications.

\section{DATA AVAILABILITY STATEMENT}

The original contributions presented in the study are included in the article/supplementary material; further inquiries can be directed to the corresponding authors.

\section{AUTHOR CONTRIBUTIONS}

JW and $\mathrm{KZ}$ conceived the experiments and supervised the project. QY and CC synthesized and characterized the samples. QY and KG performed the optical experiments and did the data analysis. $\mathrm{HD}$ and $\mathrm{YZ}$ contributed to the sample exfoliation and transfer. 
TY and WS contributed to the device design and schematic drawing. All the authors contributed to the conception and manuscript writing.

\section{FUNDING}

This research was supported by the National Natural Science Foundation of China (Grant Nos. 61922082, 61875223, and

\section{REFERENCES}

Bao, Q., Zhang, H., Wang, Y., Ni, Z., Yan, Y., Shen, Z. X., et al. (2009). Atomic-layer Graphene as a Saturable Absorber for Ultrafast Pulsed Lasers. Adv. Funct. Mater. 19 (19), 3077-3083. doi:10.1002/adfm.200901007

Chen, J., Wang, J., Yu, Q., Wang, T., Zhang, Y., Chen, C., et al. (2021). Sub-Band Gap Absorption and Optical Nonlinear Response of MnPSe3 Nanosheets for Pulse Generation in the L-Band. ACS Appl. Mater. Inter. 13 (11), 13524-13533. doi:10.1021/acsami.0c21411

Chen, M., Fang, L., Zhuang, Q., and Liu, H. (2019). Deep Learning Assessment of Myocardial Infarction from MR Image Sequences. IEEE Access 7, 5438-5446. doi:10.1109/ACCESS.2018.2889744

Cuando-Espitia, N., Bernal-Martínez, J., Torres-Cisneros, M., and May-Arrioja, D. (2019). Laser-induced Deposition of Carbon Nanotubes in Fiber Optic Tips of MMI Devices. Sensors 19 (20), 4512. doi:10.3390/s19204512

Donodin, A., Voropaev, V., Batov, D., Vlasov, D., Lazarev, V., Tarabrin, M., et al. (2020). Numerical Model of Hybrid Mode-Locked Tm-Doped All-Fibre Laser. Sci. Rep. 10 (1), 17396. doi:10.1038/s41598-020-74194-7

Geng, J., Wang, Q., Lee, Y., and Jiang, S. (2014). Development of Eye-Safe Fiber Lasers Near $2 \mu \mathrm{m}$. IEEE J. Sel. Top. Quant. 20 (5), 150-160.

Gillen, R., and Maultzsch, J. (2017). Light-Matter Interactions in Two-Dimensional Transition Metal Dichalcogenides: Dominant Excitonic Transitions in Monoand Few-Layer MoX\$_2\$ and Band Nesting. IEEE J. Select. Top. Quan. Electron. 23 (1), 219-230. doi:10.1109/JSTQE.2016.2604359

Guo, Z., Zhang, H., Lu, S., Wang, Z., Tang, S., Shao, J., et al. (2015). From Black Phosphorus to Phosphorene: Basic Solvent Exfoliation, Evolution of Raman Scattering, and Applications to Ultrafast Photonics. Adv. Funct. Mater. 25 (45), 6996-7002. doi:10.1002/adfm.201502902

Hisyam, M. B., Rusdi, M. F. M., Latiff, A. A., and Harun, S. W. (2017). Generation of Mode-Locked Ytterbium Doped Fiber Ring Laser Using Few-Layer Black Phosphorus as a Saturable Absorber. IEEE J. Select. Top. Quan. Electron. 23 (1), 39-43. doi:10.1109/JSTQE.2016.2532270

Hu, G., Albrow-Owen, T., Jin, X., Ali, A., Hu, Y., Howe, R. C. T., et al. (2017). Black Phosphorus Ink Formulation for Inkjet Printing of Optoelectronics and Photonics. Nat. Commun. 8 (1), 278. doi:10.1038/s41467-017-00358-1

Hu, Y., Qi, Z.-H., Lu, J., Chen, R., Zou, M., Chen, T., et al. (2019). Van der Waals epitaxial growth and interfacial passivation of two-dimensional singlecrystalline few-layer gray arsenic nanoflakes. Chem. Mater. 31 (12), 4524-4535. doi:10.1021/acs.chemmater.9b01151

Hu, Z., Niu, T., Guo, R., Zhang, J., Lai, M., He, J., et al. (2018). Two-dimensional Black Phosphorus: its Fabrication, Functionalization and Applications. Nanoscale 10 (46), 21575-21603. doi:10.1039/C8NR07395C

Jackson, S. D. (2012). Towards High-Power Mid-infrared Emission from a Fibre Laser. Nat. Photon 6 (7), 423-431. doi:10.1038/nphoton.2012.149

Ji, J., Song, X., Liu, J., Yan, Z., Huo, C., Zhang, S., et al. (2016). Two-dimensional antimonene single crystals grown by van der Waals epitaxy. Nat. Commun. 7 (1), 13352. doi:10.1038/ncomms13352

Kamal, C., and Ezawa, M. (2015). Arsenene: Two-Dimensional Buckled and Puckered Honeycomb Arsenic Systems. Phys. Rev. B 91 (8), 085423. doi:10.1103/PhysRevB.91.085423

Khalatpour, A., Paulsen, A. K., Deimert, C., Wasilewski, Z. R., and Hu, Q. (2021). High-power Portable Terahertz Laser Systems. Nat. Photon. 15 (1), 16-20. doi:10.1038/s41566-020-00707-5
61927813), CAS Key Laboratory of Nanodevices and Applications (21YZ03).

\section{ACKNOWLEDGMENTS}

This is a short text to acknowledge the contributions of specific colleagues, institutions, or agencies that aided the efforts of the authors.

Lee, J., Koo, J., Jhon, Y. M., and Lee, J. H. (2014). A Femtosecond Pulse Erbium Fiber Laser Incorporating a Saturable Absorber Based on Bulk-Structured Bi_2Te_3 Topological Insulator. Opt. Express 22 (5), 6165-6173. doi:10.1364/OE.22.006165

Lee, J., Koo, J., Lee, J., and Lee, J. H. (2016). End-to-End Self-Assembly of Gold Nanorods in Water Solution for Absorption Enhancement at a 1-to-2 $\mu \mathrm{m}$ Band for a Broadband Saturable Absorber. J. Lightwave Technol. 34 (22), 5250-5257. doi:10.1109/JLT.2016.2607780

Liu, H., Zheng, X.-W., Liu, M., Zhao, N., Luo, A.-P., Luo, Z.-C., et al. (2014). Femtosecond Pulse Generation from a Topological Insulator Mode-Locked Fiber Laser. Opt. Express 22 (6), 6868-6873. doi:10.1364/OE.22.006868

Ma, J., Lu, S., Guo, Z., Xu, X., Zhang, H., Tang, D., et al. (2015). Few-layer Black Phosphorus Based Saturable Absorber Mirror for Pulsed Solid-State Lasers. Opt. Express 23 (17), 22643-22648. doi:10.1364/OE.23.022643

Martinez, A., and Sun, Z. (2013). Nanotube and Graphene Saturable Absorbers for Fibre Lasers. Nat. Photon 7 (11), 842-845. doi:10.1038/nphoton.2013.304

Martov, A. G., Ergakov, D. V., Guseynov, M., Andronov, A. S., and Plekhanova, O. A. (2021). Clinical Comparison of Super Pulse Thulium Fiber Laser and HighPower Holmium Laser for Ureteral Stone Management. J. Endourology 35, 795-800. doi:10.1089/end.2020.0581

Meng, C., Yu, S.-L., Wang, H.-Q., Cao, Y., Tong, L.-M., Liu, W.-T., et al. (2015). Graphene-doped Polymer Nanofibers for Low-Threshold Nonlinear Optical Waveguiding. Light Sci. Appl. 4 (11), e348. doi:10.1038/lsa.2015.121

Moulton, P. F., Rines, G. A., Slobodtchikov, E. V., Wall, K. F., Frith, G., Samson, B., et al. (2009). Tm-doped Fiber Lasers: Fundamentals and Power Scaling. IEEE J. Select. Top. Quan. Electron. 15 (1), 85-92. doi:10.1109/jstqe.2008.2010719

Niu, X., Yi, Y., Meng, L., Shu, H., Pu, Y., and Li, X. a. (2019). Two-dimensional Phosphorene, Arsenene, and Antimonene Quantum Dots: Anomalous Sizedependent Behaviors of Optical Properties. J. Phys. Chem. C 123 (42), 25775-25780. doi:10.1021/acs.jpcc.9b04968

Pawliszewska, M., Ge, Y., Li, Z., Zhang, H., and Sotor, J. (2017). Fundamental and Harmonic Mode-Locking at $21 \mu \mathrm{m}$ with Black Phosphorus Saturable Absorber. Opt. Express 25 (15), 16916-16921. doi:10.1364/OE.25.016916

Rusdi, M. F. M., Latiff, A. A., Hanafi, E., Mahyuddin, M. B. H., Shamsudin, H., Dimyati, K., et al. (2016). Molybdenum Disulphide Tape Saturable Absorber for Mode-Locked Double-Clad Ytterbium-Doped All-Fiber Laser Generation. Chin. Phys. Lett. 33 (11), 114201. doi:10.1088/0256-307x/33/11/114201

Salam, S., Nizamani, B., Azooz, S. M., Fizza, G., Yasin, M., and Harun, S. W. (2021). Ultrafast Soliton Mode-Locked Fiber Laser at $1560 \mathrm{Nm}$ Based on Znq2 as a Saturable Absorber. Appl. Opt. 60 (11), 3149-3154. doi:10.1364/AO.418760

Shaodong, H., Chengjin, L., Haifeng, L., Jinzhang, W., Chunyu, G., Jianqun, C., et al. (2021). Ultrafast Thulium-Doped Fiber Laser Mode-Locked by Antimonides. Opt. Express 29 (9), 13722-13732. doi:10.1364/OE.421993

Shi, X., Wang, T., Wang, J., Xu, Y., Yang, Z., Yu, Q., et al. (2019). Synthesis of Black Arsenic-Phosphorus and its Application for Er-Doped Fiber Ultrashort Laser Generation. Opt. Mater. Express 9 (5), 2348-2357. doi:10.1364/OME.9.002348

Soref, R. (2015). Enabling $2 \mu \mathrm{m}$ Communications. Nat. Photon 9 (6), 358-359. doi:10.1038/nphoton.2015.87

Tang, Y., Wright, L. G., Charan, K., Wang, T., Xu, C., and Wise, F. W. (2016). Generation of Intense 100 Fs Solitons Tunable from 2 to $43 \mu \mathrm{m}$ in Fluoride Fiber. Optica 3 (9), 948-951. doi:10.1364/OPTICA.3.000948

Tuo, M., Xu, C., Mu, H., Bao, X., Wang, Y., Xiao, S., et al. (2018). Ultrathin 2D Transition Metal Carbides for Ultrafast Pulsed Fiber Lasers. ACS Photon. 5 (5), 1808-1816. doi:10.1021/acsphotonics.7b01428 
Wang, J., Wang, T., Shi, X., Wu, J., Xu, Y., Ding, X., et al. (2019a). NiPS3 Nanosheets for Passive Pulse Generation in an Er-Doped Fiber Laser. J. Mater. Chem. C 7 (46), 14625-14631. doi:10.1039/C9TC04722K

Wang, T., Jin, X., Yang, J., Wu, J., Yu, Q., Pan, Z., et al. (2019b). OxidationResistant Black Phosphorus Enable Highly Ambient-Stable Ultrafast Pulse Generation at a $2 \mu \mathrm{m}$ Tm/Ho-Doped Fiber Laser. ACS Appl. Mater. Inter. 11 (40), 36854-36862. doi:10.1021/acsami.9b12415

Wang, T., Jin, X., Yang, J., Wu, J., Yu, Q., Pan, Z., et al. (2019c). Ultra-stable Pulse Generation in Ytterbium-Doped Fiber Laser Based on Black Phosphorus. Nanoscale Adv. 1 (1), 195-202. doi:10.1039/c8na00221e

Wang, T., Yu, Q., Guo, K., Shi, X., Kan, X., Xu, Y., et al. (2021). Sb2Te3 Topological Insulator for $52 \mathrm{Nm}$ Wideband Tunable Yb-Doped Passively Q-Switched Fiber Laser. Front. Inform. Technol. Electron. Eng. 22 (3), 287-295. doi:10.1631/ FITEE.2000577

Wu, X., Shao, Y., Liu, H., Feng, Z., Wang, Y.-L., Sun, J.-T., et al. (2017). Epitaxial Growth and Air-Stability of Monolayer Antimonene on PdTe2. Adv. Mater. 29 (11), 1605407. doi:10.1002/adma.201605407

Wu, Z., and Hao, J. (2020). Electrical Transport Properties in Group-V Elemental Ultrathin 2D Layers. Npj 2d Mater. Appl. 4 (1), 4. doi:10.1038/s41699-020-0139-x

Xu, N., Li, H., Gan, Y., Chen, H., Li, W., Zhang, F., et al. (2020a). Zero-Dimensional MXene-Based Optical Devices for Ultrafast and Ultranarrow Photonics Applications. Adv. Sci. 7 (22), 2002209. doi:10.1002/advs.202002209

Xu, Y., Liu, C., Guo, C., Yu, Q., Guo, W., Lu, W., et al. (2020b). High Performance Near Infrared Photodetector Based on In-Plane Black Phosphorus P-N Homojunction. Nano Energy 70, 104518. doi:10.1016/j.nanoen.2020.104518

Xu, Y., Shi, X., Zhang, Y., Zhang, H., Zhang, Q., Huang, Z., et al. (2020c). Epitaxial Nucleation and Lateral Growth of High-Crystalline Black Phosphorus Films on Silicon. Nat. Commun. 11 (1), 1330. doi:10.1038/s41467-020-14902-Z

Yoon, J., Jo, S., Chun, I. S., Jung, I., Kim, H.-S., Meitl, M., et al. (2010). GaAs Photovoltaics and Optoelectronics Using Releasable Multilayer Epitaxial Assemblies. Nature 465 (7296), 329-333. doi:10.1038/nature09054

Zhang, C., Zhang, Y., Yuan, X., Lu, S., Zhang, J., Narayan, A., et al. (2019a). Quantum Hall Effect Based on Weyl Orbits in Cd3As2. Nature 565 (7739), 331-336. doi:10.1038/s41586-018-0798-3

Zhang, J. L., Zhao, S., Han, C., Wang, Z., Zhong, S., Sun, S., et al. (2016a). Epitaxial Growth of Single Layer Blue Phosphorus: a New Phase of Two-Dimensional Phosphorus. Nano Lett. 16 (8), 4903-4908. doi:10.1021/acs.nanolett.6b01459

Zhang, J. L., Zhao, S., Sun, S., Ding, H., Hu, J., Li, Y., et al. (2020a). Synthesis of Monolayer Blue Phosphorus Enabled by Silicon Intercalation. ACS Nano 14 (3), 3687-3695. doi:10.1021/acsnano.0c00822

Zhang, L., Huang, H., Zhang, B., Gu, M., Zhao, D., Zhao, X., et al. (2020b). Structure and Properties of Violet Phosphorus and its Phosphorene Exfoliation. Angew. Chem. Int. Ed. 59 (3), 1074-1080. doi:10.1002/anie.201912761
Zhang, M., Wu, Q., Zhang, F., Chen, L., Jin, X., Hu, Y., et al. (2019b). 2D Black Phosphorus Saturable Absorbers for Ultrafast Photonics. Adv. Opt. Mater. 7 (1), 1800224. doi:10.1002/adom.201800224

Zhang, S., Guo, S., Chen, Z., Wang, Y., Gao, H., Gómez-Herrero, J., et al. (2018). Recent Progress in 2D Group-VA Semiconductors: from Theory to experiment. Chem. Soc. Rev. 47 (3), 982-1021. doi:10.1039/C7CS00125H

Zhang, S., Xie, M., Li, F., Yan, Z., Li, Y., Kan, E., et al. (2016b). Semiconducting Group 15 Monolayers: A Broad Range of Band Gaps and High Carrier Mobilities. Angew. Chem. Int. Ed. 55 (5), 1666-1669. doi:10.1002/ anie. 201507568

Zhao, J., Liu, C., Guo, W., and Ma, J. (2017). Prediction on the Light-Assisted Exfoliation of Multilayered Arsenene by the Photo-Isomerization of Azobenzene. Nanoscale 9 (21), 7006-7011. doi:10.1039/C7NR01667K

Zhong, M., Xia, Q., Pan, L., Liu, Y., Chen, Y., Deng, H.-X., et al. (2018). Thicknessdependent Carrier Transport Characteristics of a New 2D Elemental Semiconductor: Black Arsenic. Adv. Funct. Mater. 28 (43), 1802581. doi:10.1002/adfm.201802581

Zhou, X., Feng, W., Li, F., and Yao, Y. (2017). Large Magneto-Optical Effects in Hole-Doped Blue Phosphorene and gray Arsenene. Nanoscale 9 (44), 17405-17414. doi:10.1039/C7NR05088G

Zhu, Z., Guan, J., and Tománek, D. (2015). Strain-induced Metal-Semiconductor Transition in Monolayers and Bilayers of gray Arsenic: A Computational Study. Phys. Rev. B 91 (16), 161404. doi:10.1103/PhysRevB.91.161404

Conflict of Interest: The authors declare that the research was conducted in the absence of any commercial or financial relationships that could be construed as a potential conflict of interest.

The handling editor declared a past co-authorship with one of the authors $\mathrm{KZ}$.

Publisher's Note: All claims expressed in this article are solely those of the authors and do not necessarily represent those of their affiliated organizations, or of the publisher, the editors, and the reviewers. Any product that may be evaluated in this article, or claim that may be made by its manufacturer, is not guaranteed or endorsed by the publisher.

Copyright (c) $2021 \mathrm{Yu}$, Chen, Guo, Deng, Yi, Zhang, Su, Wu and Zhang. This is an open-access article distributed under the terms of the Creative Commons Attribution License (CC BY). The use, distribution or reproduction in other forums is permitted, provided the original author(s) and the copyright owner(s) are credited and that the original publication in this journal is cited, in accordance with accepted academic practice. No use, distribution or reproduction is permitted which does not comply with these terms. 